\title{
GESTIÓN DEL CONOCIMIENTO, ACTIVIDAD CIENTÍFICA Y ENTORNOS PERSONALES DE APRENDIZAJE (PLES): UNABIBLIOMETRÍA DE LA PLE CONFERENCE
}

\author{
KNOWLEDGE MANAGEMENT, SCIENTIFIC ACTIVITY AND \\ PERSONAL LEARNING ENVIRONMENTS (PLES): A BIBLIOMETRIC \\ OF THE PLE CONFERENCE
}

\author{
María Alicia Hernández Fernández; mariaalicia.hernandez@um.es \\ Universidad de Murcia
}

\section{RESUMEN}

La PLE Conference, es una conferencia dedicada al Entorno Personal de Aprendizaje (PLE), cuyo análisis estadístico mediante el uso de la Bibliometría, sirve para evaluar la actividad científica desempeñada en relación al PLE. Con motivo de ello, la presente investigación constituye un estudio bibliométrico de la PLE Conference que tiene por objetivo primordial describir la contribución que ha realizado la PLE Conference a la literatura científica sobre el PLE, considerando para ello la producción científica generada y su consumo. En el transcurso, se ha seguido una metodología sistemática empleando indicadores bibliométricos, que ha permitido establecer conclusiones sobre el impacto y transcendencia de la PLE Conference y aportar información valiosa para optimizar la conferencia y descubrir la evolución del concepto PLE.

PALABRAS CLAVE: Entorno Personal de Aprendizaje (PLE), PLE Conference, Bibliometría, evaluación actividad científica.

\section{ABSTRACT}

The PLE Conference is a conference dedicated to Personal Learning Environment (PLE), whose statistical analysis using Bibliometrics can be used to evaluate the scientific activity carried out in relation to PLE. For this reason, the present investigation is a bibliometric study of the PLE Conference which aims primarily to describe the contribution made by the PLE Conference to scientific literature on the PLE, considering the scientific production and consumption. Throughout, it has followed a systematic methodology using bibliometric indicators, which allowed drawing conclusions about the impact and significance of the PLE Conference and providing 
valuable information to optimize the conference and discover the evolution of the PLE concept.

KEYWORDS: Personal Learning Environment (PLE), PLE Conference, Bibliometrics, scientific activity assessment.

\section{INTRODUCCIÓN}

En la actualidad, las Tecnologías de la Información y la Comunicación (TIC) se encuentran integradas en los procesos de enseñanza-aprendizaje, concibiéndose como un medio más para conseguir las grandes finalidades de la Educación. Partiendo de ésta concepción, en el campo de la Educación se incluye la disciplina denominada Tecnología Educativa (TE) cuyo ámbito de estudio según Area (2009) son: "las relaciones entre las Tecnologías de la Información y la Comunicación y la Educación" (p. 20). En la TE, el interés reside en el estudio y determinación de pedagogías acordes a ésta nueva forma de entender la educación con TIC, las cuales reciben el nombre de pedagogías emergentes y pretenden aprovechar todo el potencial que ofrecen las TIC en la educación (Adell y Castañeda, 2012), porque lo importante es "mejorar el modo en que el estudiante aprende con TIC", como apuntan Gallego y Chaves (2014, p. 2).

Como parte de éstas nuevas pedagogías, cobra importancia la idea del Entorno Personal de Aprendizaje (PLE) definido por Adell y Castañeda (2010) como “... el conjunto de herramientas, fuentes de información, conexiones y actividades que cada persona utiliza de forma asidua para aprender" (p.23). Ahora bien, cada persona utiliza su PLE para diferentes usos educativos, de tal forma que el PLE está formado por herramientas y estrategias de lectura, de reflexión y de relación (ídem, 2013). En otras palabras, para aprender cada persona cuenta con un conjunto de "sitios o servicios" de Internet que utiliza para informarse, reflexionar sobre lo aprendido y compartir sus conocimientos $y$, durante ese aprendizaje, está realizando una serie de actividades (lectura, creación de vídeos, etc.) que ponen en marcha diferentes mecanismos (síntesis, asertividad, etc.).

El hecho de que el PLE incluya la acción de compartir significa que las personas pueden ser una fuente de información y la interacción entre sujetos puede generar experiencias enriquecedoras. Éste carácter social del PLE, da lugar a la creación de las denominadas Redes Personales de Aprendizaje (PNL), que siguiendo a los anteriores autores ibídem, pueden ser de diferentes tipos: redes donde la relación se produce a través de "objetos" publicados (Slideshare, Youtube, etc.), redes donde comunicamos lo que hacemos y aprendemos (wikis, blogs, etc.) y, por último, redes que sirven para establecer relaciones con otras personas (Facebook, Twitter, etc.). Asimismo, su uso implica diferentes formas de aprender: aprender de objetos creados por los demás (vídeos, presentaciones, etc.), aprender de las actividades y experiencias de otros y, aprender interaccionando con los otros. 
La idea del PLE descrita, constituye una nueva forma de entender el proceso de enseñanza-aprendizaje con TIC centrado en el alumnado, que adquiere su importancia debido a que como indica Navas (2013) la contemplación de la optimización del PLE del alumnado por parte de los docentes, contribuye al desarrollo de la competencia de "aprender a aprender" de los discentes. Por lo tanto, el desarrollo del PLE va a facilitar el aprendizaje permanente a lo largo de la vida y, además, como afirma Salinas (2013), la gestión personal del aprendizaje. Desde estas afirmaciones, el PLE se presenta como una solución para que las personas puedan aprender de forma autónoma y autorregular su aprendizaje empleando para ello las TIC, lo cual es necesario debido a que "en un mundo cada vez más interconectado y tecnológicamente mediado, la gente tiene que actualizar continuamente sus conocimientos y habilidades, de manera que sean capaces de autodirigir sus proyectos de aprendizaje intencionales" (Väljataga \& Laanpere 2010, p.1).

En éste sentido, el PLE responde a ésta necesidad constituyéndose como "un concepto nuevo y en auge" como apuntan Gallego y Chaves (2014, p.22) que adquiere importancia en la Educación superior, la TE y la investigación. Su importancia en el mundo educativo queda plasmada en los proyectos de investigación dedicados con los que cuenta en España como es el proyecto CAPPLE (Competencias para el aprendizaje permanente basado en el uso de PLEs: análisis de los futuros profesionales y propuestas de mejora) de la Universidad de Murcia y el proyecto "Análisis y mejora de las competencias transversales universitarias en un entorno mixto e-portfolio/PLE" de la Universidad de Barcelona, entre otros. Además, en la Unión Europea son destacables los proyectos "Responsive Open Learning Environments" (ROLE) y "Personal E-Learning In Communities And Networking Spaces" (PELICANS) y los proyectos internacionales como el de Mahara en Nueva Zelanda y el del instituto de Tecnología de la información para la NRC de Canadá.

Igualmente, la importancia del PLE queda demostrada con la existencia de una conferencia internacional dedicada, la PLE Conference que marcó un punto de inflexión en el concepto de PLE, permitiendo según indican Castañeda y Adell (2013) la existencia de "un foro periódico, dedicado exclusivamente a los PLES en el que no se tratase el tema de manera periférica a la tecnología educativa, sino como un eje desde el que podrían analizarse otros temas" (p.13). Por lo tanto, la PLE Conference se erige como el espacio por excelencia para la colaboración científica y el intercambio de ideas en relación al PLE, con la finalidad de progresar en la descripción del concepto y contribuir a su evolución y, hasta el momento, han sido realizadas cinco ediciones periódicas: en 2010 en Barcelona, España; en 2011 en Southampton, Reino Unido; en 2012 en Aveiro, Portugal y Melbourne, Australia; en 2013 en Berlín, Alemania y Melbourne, Australia y en 2014 en Tallin, Estonia.

La PLE Conference se ha constituido como un motor para la actividad científica en torno al concepto de PLE que ha servido para profundizar en el concepto; por lo tanto, los artículos que la constituyen pueden considerarse a su vez motores de la evolución y difusión del concepto. En base a ésta afirmación, un estudio estadístico de la PLE Conference queda justificada para obtener valiosa información acerca de la producción 
científica sobre PLE generada y su consumo por parte de la comunidad científica y, para ello, la Bibliometría se ofrece como un método óptimo a tal fin que permite describir y evaluar la actividad científica.

La Bibliometría es definida por Pritchard (1969) como "la aplicación de métodos estadísticos y matemáticos a libros y otros medios de comunicación" (p.3). Su aplicación permite evaluar la actividad científica y, para ello, cuenta con los llamados indicadores bibliométricos definidos por Sanz y Martín (1997) como: "Los datos numéricos extraídos de los documentos que publican los científicos o que utilizan los usuarios, y que permiten el análisis de las diferentes características de su actividad científica, vinculadas, tanto a su producción como a su consumo de información" (p.46). En relación a dichos indicadores, gracias al trabajo realizado por Lascurain (2006) conocemos que cada indicador muestra un aspecto de la evaluación, aportando cada uno información valiosa para proporcionar un conocimiento multidimensional de conjunto, aunque no están exentos de presentar ciertas limitaciones como describe Sancho (1990).

Tras todo lo expuesto, considerando la importancia del PLE en la Educación y la importancia de su conferencia internacional dedicada como motor del concepto, es de recibo la realización de un estudio bibliométrico para describir cuantitativamente la actividad científica realizada sobre PLE en el contexto de la PLE Conference, como una forma de gestión de la información existente que va a permitir evaluar el presente de la conferencia y realizar valoraciones prospectivas de futuro. Además, la importancia de la presente investigación reside en la novedad que supone para la ciencia, ante la inexistencia de estudios bibliométricos en relación al PLE, al aportar una visión de la situación actual del tema desde una perspectiva diferente a las que ofrecen las revisiones bibliográficas sobre el PLE, como las ofrecidas por Gallego y Chaves (2014), Buchem, Attwell, y Torres (2011) y Chaves (2014).

\section{MÉTODO}

\subsection{Objetivos}

El objetivo general, alrededor del cual gira toda la investigación es describir la contribución que ha realizado la PLE Conference a la literatura científica sobre PLE, en base a la producción científica generada y el consumo de la misma. A su vez los objetivos específicos son conocer: la repercusión de la PLE Conference en la literatura científica sobre PLE, la producción científica sobre PLE difundida a través de la PLE Conference y la colaboración científica establecida en la PLE Conference.

\subsection{Diseño y procedimientos seguidos}

El presente trabajo es una investigación bibliométrica descriptiva que hace uso de la metodología bibliométrica sistemática sugerida por Romaní, Huamaní y González (2012) para aportar rigor a la investigación. De ésta forma, el diseño de la investigación 
ha dispuesto de una serie de pasos sistemáticos que quedan reflejados en la siguiente gráfica:

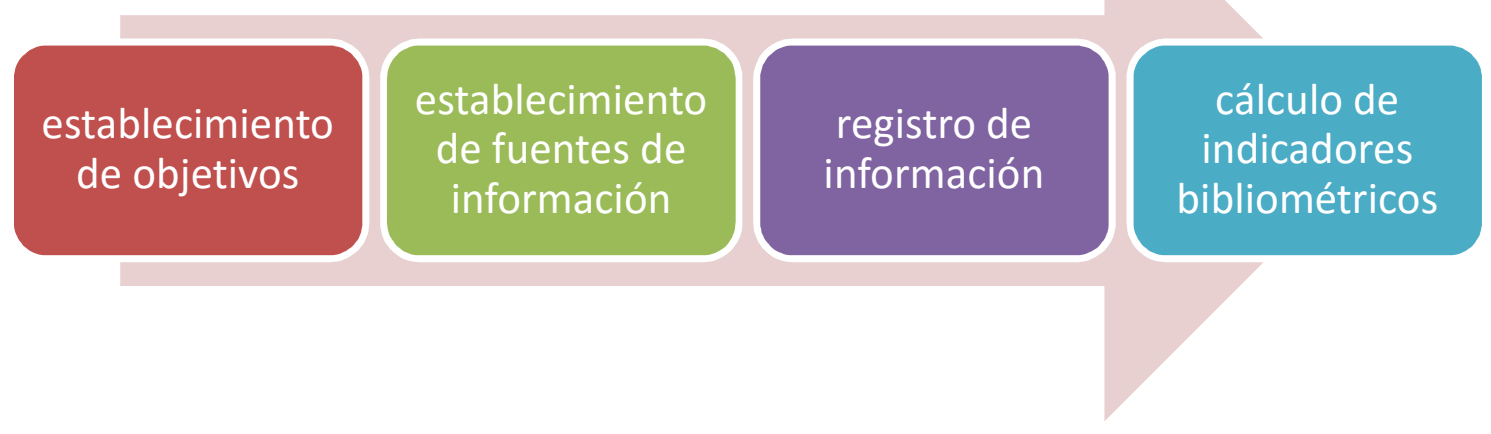

Gráfico 1: Pasos sistemáticos planteados en el diseño de la investigación, basados en la metodología bibliométrica propuesta por Romaní et al. (2012).

\section{Paso 1: Planteamiento de objetivos de investigación}

Los objetivos de la investigación son los definidos en el apartado anterior, los cuales están orientados a describir en qué medida la PLE Conference ha repercutido en la literatura sobre el PLE, ha fomentado la colaboración internacional y ha facilitado el consumo de información en relación al concepto.

\section{Paso 2: Establecimiento de fuentes de información}

Como fuentes de información fueron seleccionados los artículos aceptados que aparecen en las actas de las distintas PLE Conferences y los artículos derivados de los mismos (con diferente título) que han sido publicados en ediciones especiales de revistas internacionales, que son las siguientes:

\footnotetext{
* Digital Education Review, 182010

* Digital Education Review, 20, 2011

* International Journal of Virtual and Personal Learning Environments, 2(4), 2011

* International Journal of Virtual and Personal Learning Environments, 4(4), 2013

* elearning Papers n.o 35 • Noviembre 2013

* Journal of Literacy and Technology. Volume 15, Number 2: June 2014
}

Para la obtención de información, se optó por seleccionar el motor de búsqueda Google Académico especializado en artículos científicos, que permite comprobar la disponibilidad de los artículos y muestra la cantidad de citas recibidas y el idioma de las mismas. 


\section{Paso 3: Registro de información}

El registro de la información empleando la técnica de la observación, fue realizado accediendo a las bases de datos a las que redirigen o a los documentos directamente, empleando los criterios que se muestran a continuación:

* Disponibilidad: posibilidad de acceso por parte de los usuarios al documento escrito.

* Idioma: lengua en la cual han sido escritos los artículos objeto de estudio.

* Citación e idioma: número de artículos posteriores que han citado en sus bibliografías los artículos objeto de estudio y el idioma en el cual han sido publicados los artículos que citan.

* Autocitación de los autores: número de veces que los autores de los artículos objeto de estudio han citado sus artículos en otros creados posteriormente.

* Autocitación a la Conferencia PLE: número de artículos que han sido citados en trabajos aceptados en posteriores ediciones de la Conferencia PLE.

* Procedencia: países de referencia de los autores.

* Autoría: número de artículos que han sido creados por un único autor o varios.

* Colaboración: número de autores que han elaborado artículos en colaboración con otros autores afiliados a instituciones diferentes a la propia, a nivel de país y mundial.

Por otro lado, para el registro y tratamiento de la información, fue empleado el programa informático Excel integrado en Microsoft Office 2007, creando documentos para cada edición de la conferencia y la PLE Conference en general, más un documento adicional para los autores, asignando campos en base a los criterios marcados en las celdas de cada documento.

\section{Paso 4: Cálculo de indicadores bibliométricos}

El empleo de indicadores bibliométricos en la investigación, sirve según Ardanuy (2012) para: "expresar cuantitativamente las características bibliográficas del conjunto de documentos estudiado así como las relaciones existentes entre estas características" (p.16); por lo tanto, su uso nos van a permitir el análisis de la actividad científica mostrada en la PLE Conference, en lo referente tanto a la producción como al consumo de la literatura sobre PLE.

Ante la gran serie de propuestas de clasificación existentes sobre indicadores bibliométricos, se estimó oportuno utilizar la clasificación ofrecida por Ardanuy (2012), contemplando los indicadores personales, de producción, de visibilidad e impacto, de colaboración y de forma y contenido. Sin embargo, por la naturaleza de investigación se ha estimado conveniente también incluir la utilización de los indicadores de circulación mencionados por Portillo, Portillo y Morillo (2011).

Cada uno de los indicadores ofrece información desde una perspectiva diferente tal como indica la autora Lascurain (2006), lo cual hace necesario relacionarlos para poder adquirir una visión holística. Con motivo de ello, se ha incluido como parte del estudio 
la comparación de sus valores, con la finalidad de detectar relaciones correlativas significativas; es decir, muestras de la existencia de reciprocidad entre los indicadores, sin que ello suponga una relación causal.

\section{Indicadores personales}

Los indicadores personales se ocupan de las características de los autores del estudio. En nuestro caso es planteado el uso de indicadores personales para establecer la cantidad de autores y de países que han participado activamente.

\section{Indicadores de producción}

Los indicadores de producción aportan información respecto a los artículos, siendo empleados en la presente investigación para clasificar a los autores en función de su producción y calcular los siguientes índices: índice de productividad de los autores e índice de transitoriedad.

El índice de productividad personal de los autores, midiéndose a través del número de publicaciones por investigador, sigue la Ley de Lotka definida como el logaritmo decimal del número de artículos realizados.

$\mathrm{IP}=\log \mathrm{N}$

Donde: IP es el indicador de productividad personal y $\mathrm{N}$ es el número de artículos.

El cálculo de éste índice permite clasificar los autores en tres tipos según su productividad:

* Grandes productores 1 2IP (10 o más trabajos e índice de productividad igual o mayor que 1).

* Productores intermedios $0<\mathrm{IP}<1$ (entre 2 y 9 trabajos e índice de productividad mayor que 0 y menor que 1 ).

- Productores transitorios IP = 0 (con un sólo trabajo y un índice de productividad igual a 0).

Complementariamente, el cálculo de éste índice permite a su vez conocer el índice de transitoriedad entendido como el porcentaje de autores transitorios (con un sólo trabajo).

\section{Indicadores de visibilidad e impacto}

Los indicadores de visibilidad e impacto, miden la influencia de los autores y de los trabajos publicados mediante el análisis de citas.

Como parte de éstos indicadores, se ha determinado calcular el índice de impacto descrito por Escorcia (2008) como un índice fruto del cociente entre el número de artículos citados y los publicados. Asimismo, se establecido la creación de un ranking 
en función de los artículos más citados y el cálculo del índice de autocitación entendido como el cociente entre el total de autocitaciones y el total de citas multiplicado por cien, cuyo resultado es un porcentaje. Respecto a las autocitaciones, cabe destacar que se ha procedido a considerar como tales tanto el número de citas que los autores han realizado a sus propios trabajos, como el número de citas recibidas de artículos que han sido aceptados en posteriores Conferencias PLE.

Finalmente, se ha incluido la cuantificación de los idiomas de los artículos que citan, con la pretensión de cuantificar la expansión lingüística.

\section{Indicadores de colaboración}

Los indicadores de colaboración miden las relaciones entre los autores y para la presente investigación, su uso se ha determinado para establecer las cantidades de la autoría, referidas a los trabajos que han sido realizados en colaboración.

\section{Indicadores de forma y contenido}

Los indicadores de forma y contenido, aportan información sobre cómo son los artículos estudiados, siendo empleados en ésta investigación para conocer el idioma en el que han sido escritos los artículos objeto de estudio.

\section{Indicadores de circulación}

Por último, los indicadores de circulación entendidos como la forma de transmisión de la información para el consumo son utilizados en ésta investigación para conocer la presencia de los artículos en el motor de búsqueda utilizado. En relación a éstos indicadores, se ha planteado establecer la cantidad de artículos no disponibles al público y calcular el índice de circulación, entendiendo éste como el cociente entre el número de artículos circulantes y el total de artículos, cuya función es indicar la difusión que han presentado los artículos y sus posibilidades de contribuir al desarrollo de la ciencia.

\section{RESULTADOS Y DISCUSIÓN}

Cada uno de los indicadores empleados ha ofrecido una serie de resultados que, diferenciadamente, son los siguientes:

\section{Indicadores personales}

Los indicadores personales muestran que han expuesto sus trabajos un total de 321 autores que provienen de 32 países diferentes. Aunque no ha sido posible conocer los países de procedencia de 16 de ellos, el origen de los 305 autores restantes nos permite conocer la cantidad de autores que han contribuido en la PLE Conference de cada país, tal como se muestra a continuación: 


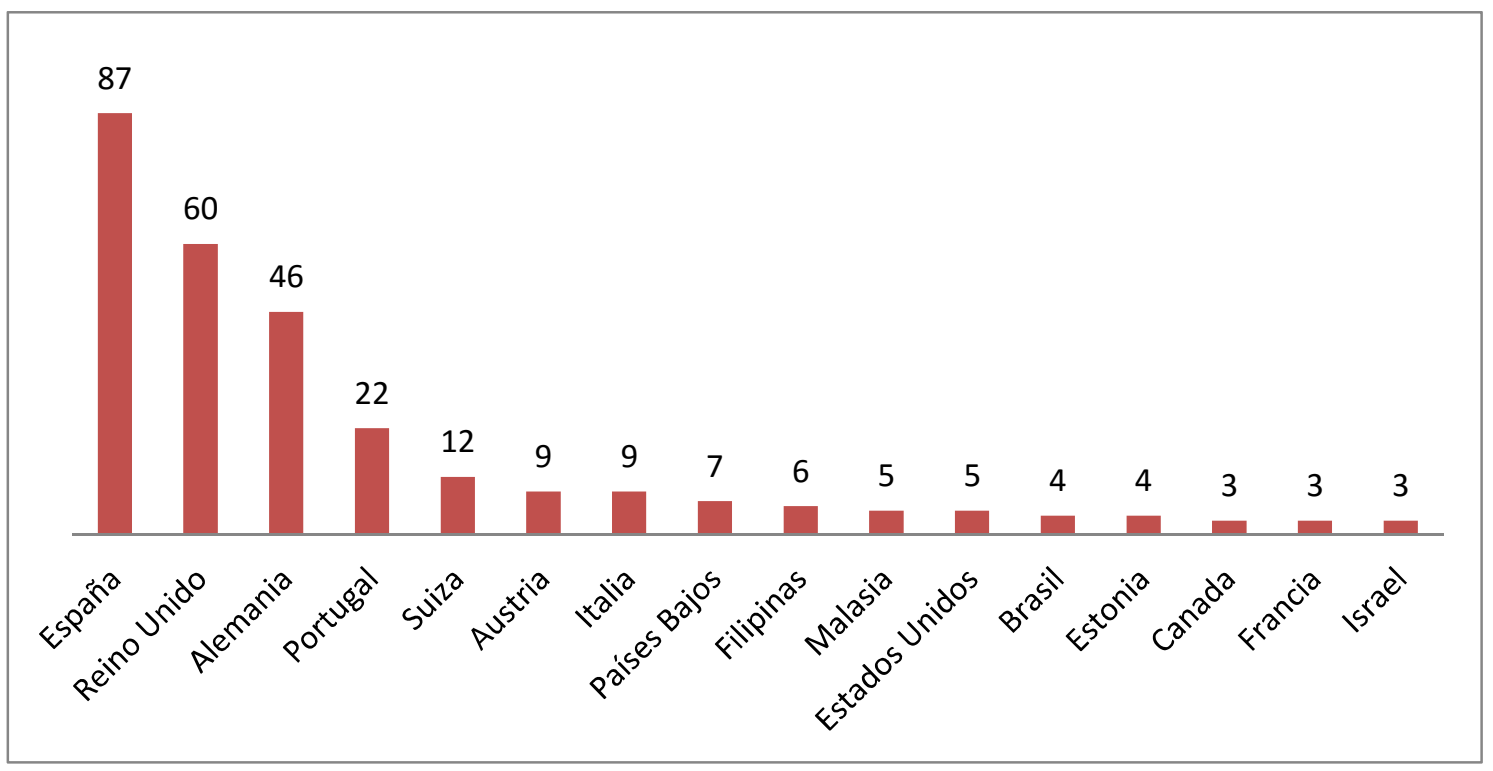

Gráfico 2: Número de autores de cada país que han contribuido a la PLE Conference.

Como se puede apreciar, en la PLE Conference los países que cuentan con un mayor número de autores contribuyentes son España, el Reino Unido y Alemania. Ahora bien, los países de la gráfica no son los únicos que cuentan con representación en la PLE Conference, sino que también otros países se han visto implicados aunque sus autores hayan contribuido en menor medida. Dichos países son: Finlandia, Pakistán, Rumanía, Suecia, Andorra, Australia, Bélgica, Bulgaria, China, Dinamarca, Grecia, Irán, Irlanda, Letonia, Méjico y Nueva Zelanda.

\section{Indicadores de producción y de contenido y forma}

Empleando los indicadores de producción para la PLE Conference, en relación al volumen de la producción científica podemos afirmar que han sido realizados un total de 196 artículos (181 aceptados en la conferencia y 15 derivados publicados en revistas) y del total de los mismos, considerando los indicadores de contenido y forma, el $98,46 \%$ se han escrito en inglés y el $1,54 \%$ en español. La relación de artículos correspondientes a cada edición de la PLE Conference, queda recogida en el siguiente gráfico:

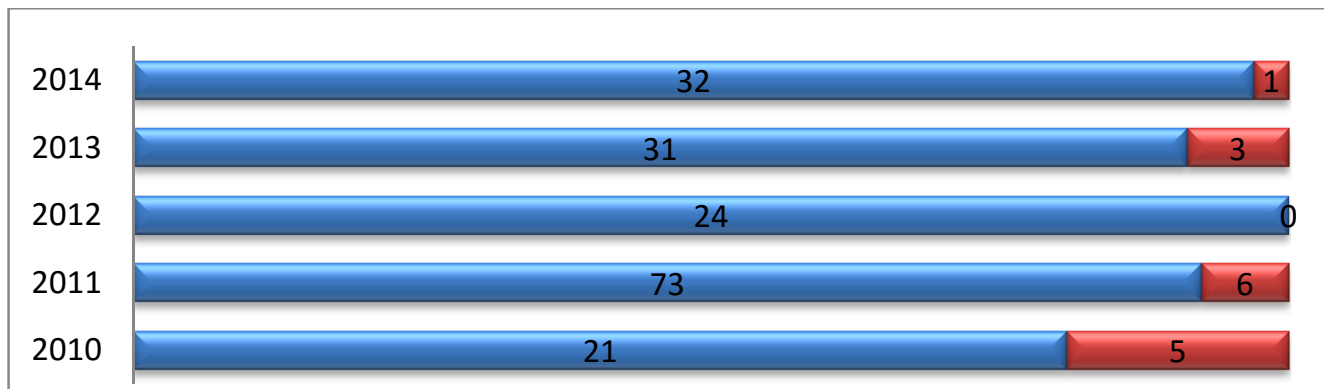

$\square$ artículos aceptados en la PLE Conference $\square$ artículos derivados en revistas

Gráfico 3: Cantidad de artículos por año, diferenciados según su procedencia. 
Como se aprecia en la gráfica, todas las ediciones han generado una cantidad equiparable de artículos, a excepción de la PLE Conference de 2011 que se constituye como la edición que mayor cantidad de artículos ha presentado.

En cuanto a los índices de productividad, el índice de productividad personal de los autores nos ha permitido clasificarlos en función de la cantidad de publicaciones que han realizado como se muestra a continuación:

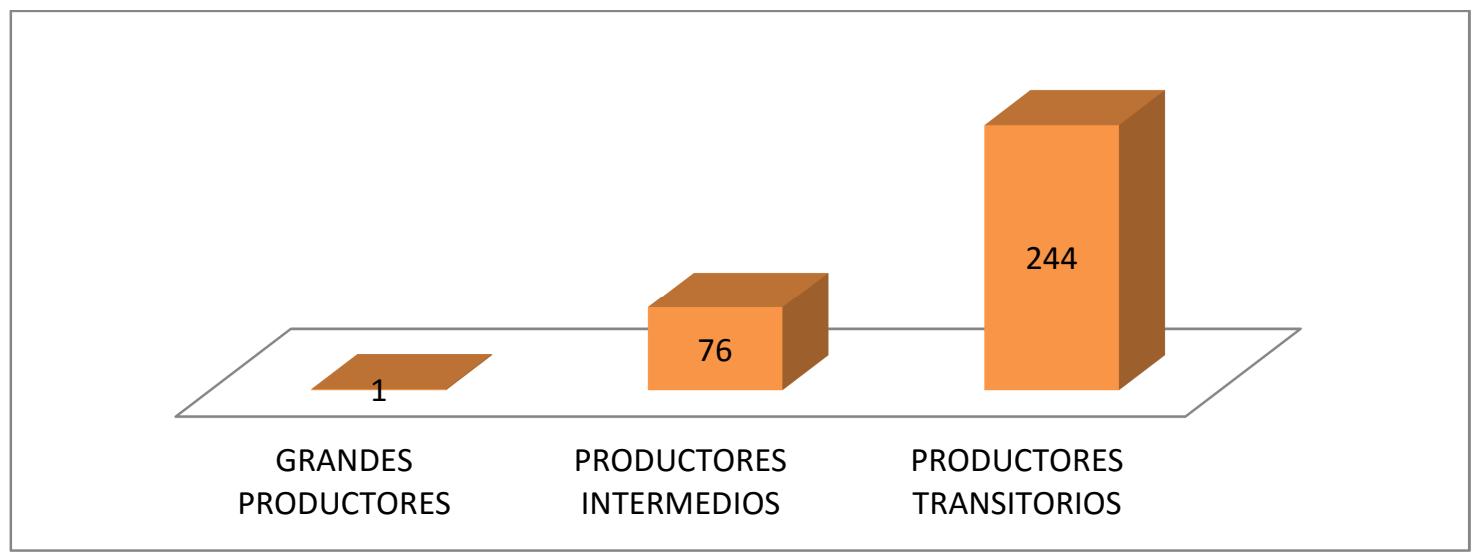

Gráfica 4: Clasificación de los autores en función del número de artículos elaborados.

Concretamente, los autores incluidos en el grupo de "grandes productores" han realizado diez o más publicaciones (con un índice de productividad igual a 1), los "productores intermedios" han contribuido con un número de artículos entre dos y nueve (con índice de productividad menor de $1 \mathrm{y}$ mayor de 0) $\mathrm{y}$, finalmente, los productores transitorios únicamente han publicado un artículo (con un índice de productividad igual a 0). En base al número de autores transitorios, el índice de transitoriedad de la PLE Conference es del 76\%.

\section{Indicadores de visibilidad e impacto}

Respecto al uso de los indicadores de visibilidad e impacto, el total de citas de la PLE Conference es de 605 citas que han sido realizadas a 92 de los 196 artículos generados; por lo tanto, han sido citados el 46,93\% de los artículos totales. La evolución del porcentaje de artículos citados que ha experimentado la PLE Conference a lo largo de sus sucesivas ediciones es la siguiente:

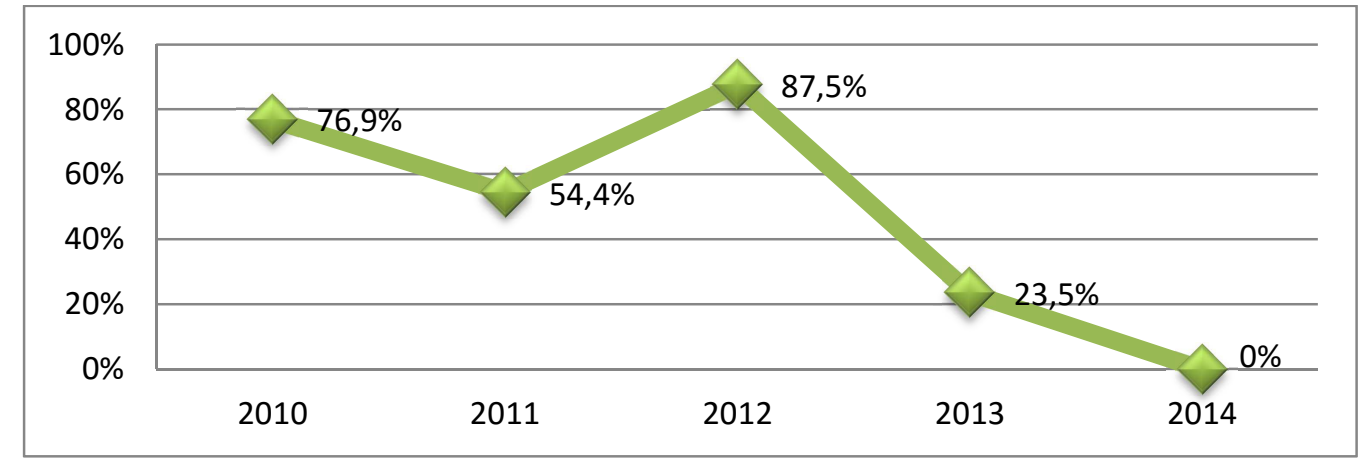

Gráfica 5: Porcentaje de artículos citados en cada edición de la PLE Conference. 
La gráfica presentada muestra que la conferencia de 2012 es la que cuenta con un mayor número de artículos citados (87,5\%), a diferencia de la PLE Conference de 2014 cuyos artículos no han recibido citaciones y la conferencia de 2013, donde sólo han sido citados el $23,53 \%$ de los artículos.

En cuanto al índice de impacto del conjunto de la PLE Conference, éste es de 0,47, habiendo experimentado dicho índice la siguiente evolución:

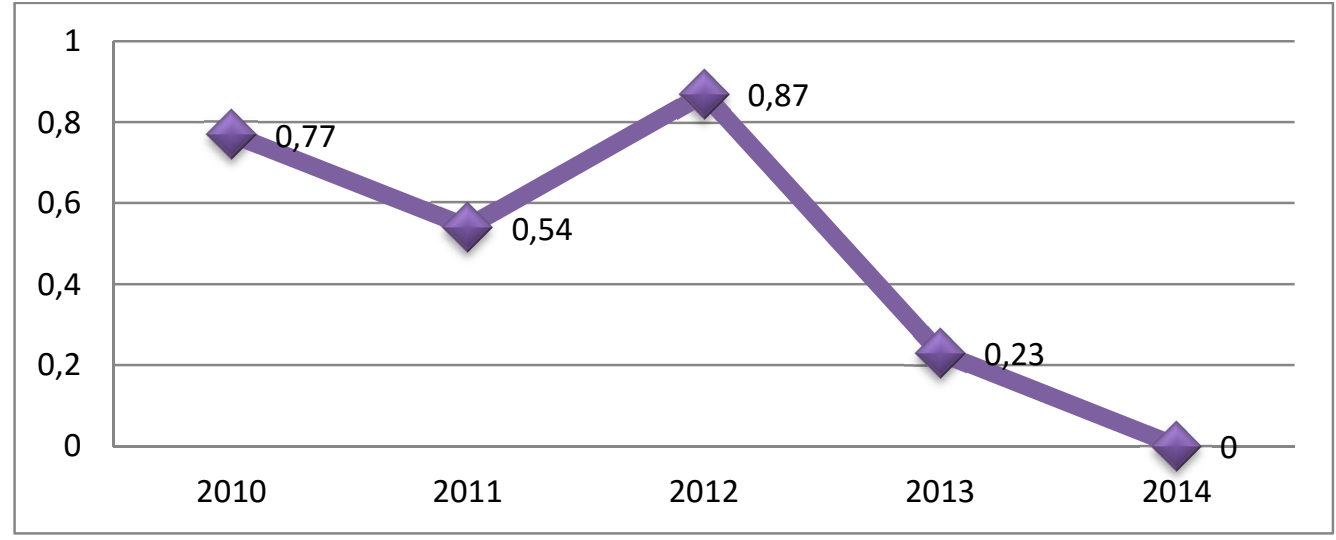

Gráfico 6: Índice de impacto de las diferentes ediciones de la PLE Conference.

Los datos expuestos en la gráfica, ponen de manifiesto que el mayor índice de impacto ha sido obtenido por la PLE Conference de $2012(0,87)$, al contrario que la conferencia de 2013 que cuenta con un índice de impacto de 0,23 y la conferencia de 2014 con un índice de impacto igual a 0.

Considerando las autocitaciones, la PLE Conference presenta un total de 173 autocitaciones ( 127 realizadas por los autores y 46 a la conferencia), luego el índice de autocitación es del $26,86 \%$. La evolución de éste índice a lo largo de las ediciones es la siguiente:

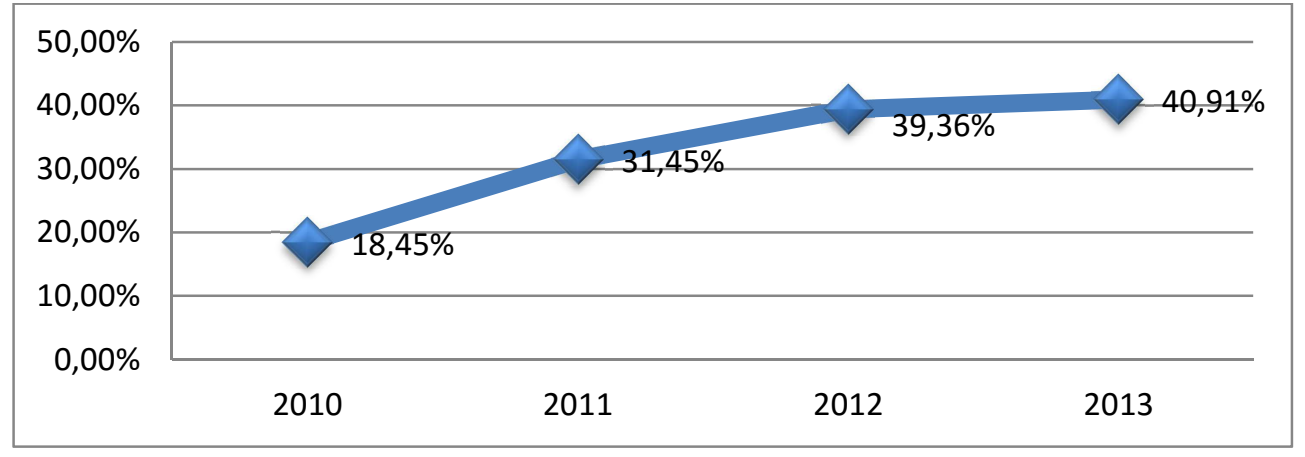

Gráfico 7: Índice de autocitación de las cuatro primeras ediciones de la PLE Conference.

El gráfico muestra una tendencia al aumento de las autocitaciones a lo largo de las cuatro primeras ediciones de la PLE Conference (la conferencia de 2014 no ha podido considerarse por no presentar citas).

En relación al idioma de las citas, la representación de las mismas para la PLE Conference es la siguiente: 


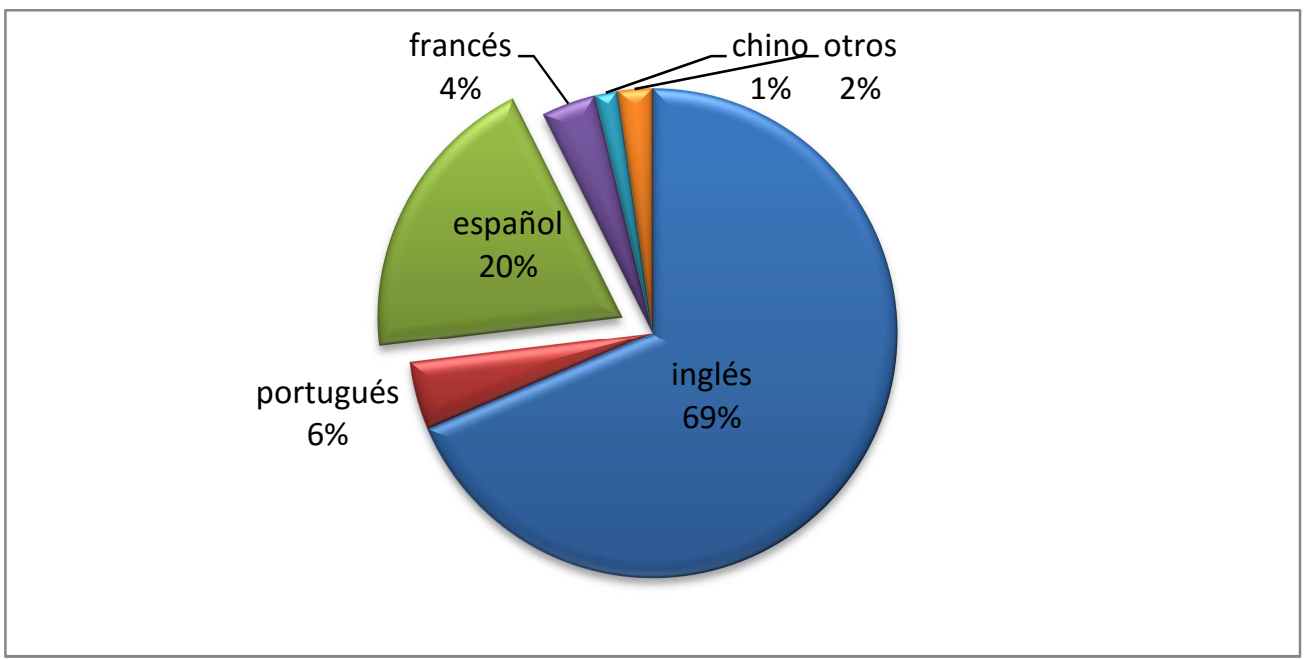

Gráfica 8: Idiomas empleados en las citas totales de la PLE Conference.

Como se puede visualizar en la gráfica, el idioma inglés ha sido el más comúnmente utilizado en los artículos que citan; no obstante, es de resaltar la gran variedad de idiomas empleados por sus respectivos autores.

Como último punto de los indicadores de visibilidad e impacto, se ha establecido un ranking con los artículos más citados del conjunto de la PLE Conference, que suman entre ellos más de un tercio del total de las citas, quedando recogidos en la siguiente tabla:

\section{REFERENCIAS BIBLIOGRÁFICAS}

NN DE CITAS

Fiedler, S. y Väljataga, T. (2010): Personal learning environments: concept or technology?. Proceeding in the PLE Conference 2010

Buchem, I., Attwell, G., y Torres, R. (2011): Understanding personal learning environments: Literature review and synthesis through the activity theory lens. Proceeding in the PLE Conference 2011

Castañeda, L. y Soto, J. (2010): Building Personal Learning Environments by Using and Mixing ICT Tools in a Professional Way. Digital Education Review, 18

Attwell, G. (2010): Supporting personal learning in the workplace. Proceeding in the PLE Conference 2010

Ferguson, R., Buckingham, S. y Deakin, R. (2011). Enquiryblogger: using widgets to support awareness and reflection in a PLE setting. Proceeding in the PLE Conference 2011

Tabla 1: Artículos más citados del conjunto de la PLE Conference.

\section{Indicadores de colaboración}

Los indicadores de colaboración respecto a la autoría de la producción científica permiten afirmar que la cantidad de trabajos de autoría asciende a 30 artículos y los trabajos de coautoría son un total de 166 artículos; por consiguiente, el 15,30\% del total de los artículos son autorías y el $84,70 \%$ son coautorías. 


\section{Indicadores de circulación}

Para finalizar el análisis, los indicadores de circulación muestran que del total de artículos, 135 se encuentran disponibles en contraposición a los 61 que no son "circulantes"; de tal modo que, el $68,87 \%$ de los artículos está disponible y el 31,12\% no permite su lectura a través de Google. Por lo mismo decimos que, el índice de circulación del conjunto de la PLE Conference es de 0,69. La evolución del mencionado índice en el transcurso de las ediciones de la PLE Conference, queda representado en la gráfica que se expone a continuación:

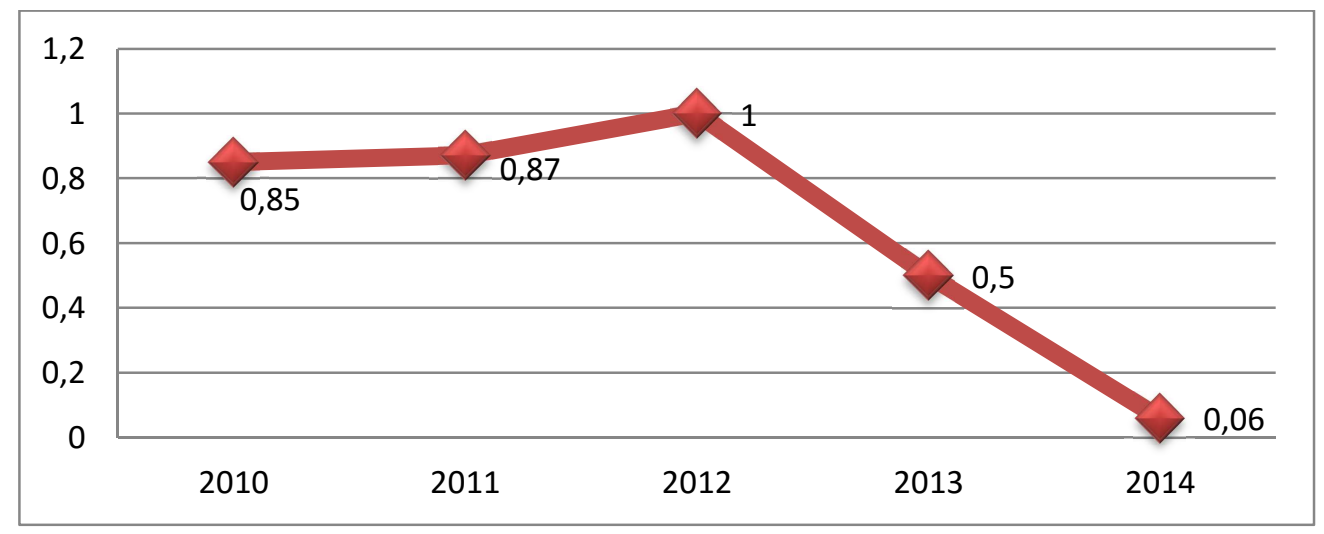

Gráfica 11: Índice de circulación de cada edición de la PLE Conference.

Tal como muestra la gráfica, la PLE Conference de 2012 es la única edición de la conferencia que presenta todos sus artículos disponibles públicamente en Google y, contrariamente, la edición de 2014 ha sido la edición que ha presentado una circulación de la producción científica más baja, prácticamente nula.

\section{CONCLUSIONES}

Fruto de la comparación entre las diferentes ediciones y el análisis de los datos de los datos generales se concluye que:

- Existe una tendencia manifiesta a la coautoría, lo cual coincide con la afirmación de Gallego y Chaves (2014) a éste respecto. Asimismo, se muestra una tendencia a la transitoriedad de los autores.

- Los datos muestran que el país que mayor implicación ha denotado en la PLE Conference es España, lo cual es coherente con el hecho de que sea el país donde tuvo su origen la primera conferencia dedicada al PLE. Asimismo, los datos también ponen de manifiesto que han estado involucrados en la PLE Conference un número significativo de países a nivel internacional, que denota la transcendencia mundial de la conferencia.

Por otro lado, la puesta en relación de los indicadores bibliométricos muestra la existencia de relaciones correlativas significativas entre: 
- La disponibilidad de los artículos y el impacto. Los datos muestran que una mayor disponibilidad de los artículos se corresponde con un mayor impacto de los mismos, lo cual concuerda con la afirmación de Melero (2005) a éste respecto.

- La cantidad, la calidad y el impacto. El aumento de la cantidad de los artículos aceptados por edición guarda relación con un menor reconocimiento mostrado en las citas, lo cual podría tener una explicación plausible en el hecho de que un aumento de la cantidad haya generado un detrimento de la calidad de los artículos y, por tanto, un menor impacto. No obstante, no es función de la Bibliometría valorar la calidad de la producción científica, para ello serían necesarios la realización de otro tipo de estudios posteriores.

Como consecuencia de las correlaciones significativas establecidas, es posible hacer una prospectiva de futuro y establecer una serie de propuestas discutibles para la optimización de la PLE Conference en particular, y las conferencias y congresos en general, gracias a las aportaciones de la Bibliometría.

A éste respecto, en una primera línea podría ser recomendable que desde las conferencias y congresos se procurase la disponibilidad de las producciones científicas en la red sin restricciones, para favorecer el consumo por parte de la comunidad científica, con el fin de que puedan contribuir al desarrollo de la ciencia. Asimismo, en relación a la cantidad versus calidad, podrían emplearse ciertas pautas en la selección de los artículos y, a éste respecto, es de recibo destacar las sugerencias ofrecidas por Miñana (1999) en su trabajo "Comunicaciones científicas a congresos científicos: algunas propuestas de mejora", donde se ofrece al lector propuestas para la selección de artículos, como es el hecho de realizar una segunda revisión de las comunicaciones aceptadas, con el fin de clasificarlas en grupos atendiendo a criterios de calidad metodológica, pertinencia y originalidad.

Concretamente, en el caso de la PLE Conference de 2011, ésta acción hubiese permitido crear una selección de artículos de entre los 73 aceptados que, a juicio del comité científico, serían los de mayor calidad. De ésta forma, la calidad de los artículos seleccionados hubiese quedado asegurada al lector, lo cual podría haber repercutido positivamente en un mayor impacto de los mismos en la comunidad científica.

\section{REFERENCIAS}

ADELL, J. Y CASTAÑEDA, L. (2010). “Los Entornos Personales de Aprendizaje (PLEs): una nueva manera de entender el aprendizaje". En Roig Vila, R. y Fiorucci, M. (Eds.) Claves para la investigación en innovación y calidad educativas. La integración de las Tecnologías de la Información y la Comunicación y la Interculturalidad en las aulas. Stumenti di ricerca per l'innovaziones e la qualità in ámbito educativo. La Tecnologie dell'informazione e della Comunicaziones e l'interculturalità nella scuola. Alcoy: Marfil - Roma TRE Universita degli studi. Recuperado de: http://hdl.handle.net/10201/17247 
ADELL, J. Y CASTAÑEDA, L. (2012). Tecnologías emergentes, ¿pedagogías emergentes? En J. Hernández, M. Pennesi, D. Sobrino y A. Vázquez (coord.). Tendencias emergentes en educación con TIC. Barcelona: Asociación Espiral, Educación y Tecnología.

ARDANUY, J. (2012). Breve introducción a la bibliometría. Departament de Biblioteconomia i Documentació, Universitat de Barcelona.

AREA MOREIRA, M. (2009). Introducción a la tecnología educativa: manual electrónico. Recuperado de: http://bibliotecadigital.org/handle/001/415

BUCHEM, I., ATTWELL, G., Y TORRES, R. (2011). Understanding personal learning environments: Literature review and synthesis through the activity theory lens. Proceeding in the PLE Conference 2010. Recuperado de: http://journal.webscience.org/658/

CASTAÑEDA, L. Y ADELL, J. (2013). La anatomía de los PLEs. En L. Castañeda y J. Adell (Eds.), Entornos Personales de Aprendizaje: Claves para el ecosistema educativo en red (pp. 11-27). Alcoy: Marfil. Recuperado de: http://digitum.um.es/xmlui/bitstream/10201/30408/1/capitulo1.pdf

CHÁVEZ, E. (2014). Investigación educativa sobre autogestión en los Entornos Personales de Aprendizaje (PLE): una revisión de la literatura. EDMETIC, Revista de Educación Mediática y TIC, 3(2), 114-134. Recuperado de: https://www.uco.es/ojs/index.php/edmetic/article/view/2892

ESCORCIA, T. A. (2008). El análisis bibliométrico como herramienta para el seguimiento de publicaciones científicas, tesis y trabajos de grado. Pontificia Universidad Javeriana. http://javeriana.edu.co/biblos/tesis/ciencias/tesis209.pdf

GALLEGO, M.J. Y CHAVES, E. (2014). Tendencias en estudios sobre entornos personales de aprendizaje (Personal Learning Environments -PLE-). EDUTEC, Revista Electrónica de Tecnología Educativa, 49. Recuperado de: http://edutec.rediris.es/Revelec2/Revelec49/n49_Gallego_Chaves.html

LASCURAIN, M.L. (2006). La evaluación de la actividad científica mediante indicadores bibliométricos. Bibliotecas: Revista de la Escuela de Bibliotecología, Documentación e Información, 24(1 y 2), 9-26.Recuperado de: http://www.revistas.una.ac.cr/index.php/bibliotecas/article/view/429

MELERO, R. (2005). Acceso abierto a las publicaciones científicas: definición, recursos, copyright e impacto. El profesional de la información, 15(4), 255-66. Recuperado de: http://eprints.rclis.org/6571

MIÑANA, J. S. (1999). Comunicaciones científicas a congresos científicos: algunas propuestas de mejora. Aten Primaria, 23, 371-375. 
NAVAS, E. E. (2013). Los entornos personales de aprendizaje en el marco de la educación permanente. EDMETIC, Revista de Educación Mediática y TIC, 2(1), 94-110.

PORTILLO, D.P., PORTILLO, L., Y MORILLO, E.C. (2011). Indicadores de productividad, colaboración y circulación de la investigación. Caso: Escuela de Bibliotecología y Archivología de Luz. Documentación de las Ciencias de la Información, 34, 291306.

de: http://revistas.ucm.es/index.php/DCIN/article/view/36459

PRITCHARD, A., Y WITTIG, G. R. (1981). Bibliometrics. England: AllM Books.

ROMANÍ, F., HUAMANÍ, C., Y GONZÁLEZ, G. (2012). Estudios bibliométricos como línea de investigación en las ciencias biomédicas: una aproximación para el pregrado. Ciencia e Investigación Medico Estudiantil Latinoamericana, 16(1). Recuperado de: http://www.cimel.felsocem.net/index.php/CIMEL/article/viewArticle/187

SALINAS, J. (2013). Enseñanza flexible y aprendizaje abierto, fundamentos clave de los PLEs. En Castañeda, L. y Adell, J. (eds.). (2013). Entornos personales de aprendizaje: claves para el ecosistema educativo en red. Alcoy: Marfil.

SANCHO, R. (1990). Indicadores bibliométricos utilizados en la evaluación de la ciencia y la tecnología. Revisión bibliográfica. Revista española de documentación científica, 13(3-4), 77-106. Recuperado de: http://www.temarium.com/serlibre/recursos/pdf/79059.Inteligencia\%20Comp etitiva.Lecturas.pdf\#page $=77$

SANZ, E. Y MARTíN, C. (1997). Técnicas bibliométricas aplicadas a las estudios de usuarios. Revista general de información y documentación, 7(2), Recuperado de:

http://revistas.ucm.es/index.php/RGID/article/viewFile/RGID9797220041A/108 78

VÄLATAAGA, T., Y LAANPERE, M. (2010). Learner control and personal learning environment: a challenge for instructional design. Interactive Learning Environments, 18(3), 277-291. Recuperado de: http://www.tandfonline.com/doi/abs/10.1080/10494820.2010.500546

Para citar este artículo:

Hernández, M. A. (2016). Gestión del conocimiento, actividad científica y entornos personales de aprendizaje (ples): una bibliometría de la ple conference. EDUTEC, Revista Electrónica de Tecnología Educativa, 55. Recuperado el dd/mm/aa de http://www.edutec.es/revista 